Article

\title{
Asymptotic Separation of Solutions to Fractional Stochastic Multi-Term Differential Equations
}

\author{
Arzu Ahmadova *(D) and Nazim I. Mahmudov (D) \\ Department of Mathematics, Eastern Mediterranean University, Famagusta 99628, Turkey; \\ nazim.mahmudov@emu.edu.tr \\ * Correspondence: arzu.ahmadova@emu.edu.tr
}

\begin{abstract}
In this paper, we study the exact asymptotic separation rate of two distinct solutions of Caputo stochastic multi-term differential equations (Caputo SMTDEs). Our goal in this paper is to establish results of the global existence and uniqueness and continuity dependence of the initial values of the solutions to Caputo SMTDEs with non-permutable matrices of order $\alpha \in\left(\frac{1}{2}, 1\right)$ and $\beta \in(0,1)$ whose coefficients satisfy a standard Lipschitz condition. For this class of systems, we then show the asymptotic separation property between two different solutions of Caputo SMTDEs with a more general condition based on $\lambda$. Furthermore, the asymptotic separation rate for the two distinct mild solutions reveals that our asymptotic results are general.
\end{abstract}

Keywords: asymptotic separation; Caputo stochastic multi-term differential equations; existence and uniqueness; continuous dependence on initial conditions; non-permutable matrices

Citation: Ahmadova, A.;

Mahmudov, N.I. Asymptotic

Separation of Solutions to Fractional Stochastic Multi-Term Differential

Equations. Fractal Fract. 2021, 5, 256.

https://doi.org/10.3390/

fractalfract5040256

Academic Editor: Burcu Gürbüz

Received: 3 November 2021

Accepted: 2 December 2021

Published: 4 December 2021

Publisher's Note: MDPI stays neutral with regard to jurisdictional claims in published maps and institutional affiliations.

Copyright: (c) 2021 by the authors. Licensee MDPI, Basel, Switzerland. This article is an open access article distributed under the terms and conditions of the Creative Commons Attribution (CC BY) license (https:// creativecommons.org/licenses/by/ $4.0 /)$.

\section{Introduction}

Over the years, many results on the theory and applications of stochastic differential equations (SDEs) have been studied [1,2]. In physics, SDEs have wide applicability ranging from molecular dynamics to neurodynamics and to the dynamics of astrophysical objects [3]. More specifically, SDEs describe all dynamical systems, in which quantum effects are either unimportant or can be taken into account as perturbations. SDEs can be viewed as a generalization of the dynamical systems theory to models with noise. This is an important generalization because real systems cannot be completely isolated from their environments and for this reason always experience external stochastic influence [4]. SDEs are also used to model various phenomena such as unstable stock prices or physical systems subject to thermal fluctuations [5]. In particular, fractional stochastic differential equations (FSDEs), which are a generalisation of differential equations using fractional and stochastic calculus, are becoming more popular due to their applications in modeling and financial mathematics. The nonlinear systems of FDEs have been studied from various points of view: applications to population dynamics, optimal pricing in economics, and recent COVID-19 epidemics. Recently, FSDEs have been extensively used for modeling mathematical problems in finance [6,7], dynamics of complex systems in engineering [8] and other areas [9,10]. Most results on fractional stochastic dynamical systems are limited to proving the existence and uniqueness of mild solutions using the fixed point theorem. For instance, the authors in [11] studied existence and uniqueness results for a class of fractional stochastic neutral differential equations using fixed point approach and Carathéodory conditions. Rodkina [12] proved existence and uniqueness of solution of stochastic differential equations with heredity. Existence and uniqueness and asymptotic behavior of mild solutions to stochastic functional differential equations in Hilbert spaces have been considered by Taniguchi et al. [13], and local and global existence for mild solutions of stochastic differential equations was studied by Barbu [14]. One can refer different types of stability studies for FSDEs, e.g., asymptotic stability of impulsive stochastic partial differential equations with infinite delays in [15], controllability and 
stability of fractional stochastic functional systems driven by Rosenblatt process in [16], an exponential stability of SDEs in [17].

Using fractional derivatives instead of integer-order derivatives allows for the modelling of a wider variety of behaviours. Fractional stochastic Langevin equations (FSLEs) is an untreated topic in the present literature, and worthy of investigation because many of the stochastic problems in fluctuating media can be described by the Langevin Equation $[18,19]$. However, sometimes, SDEs involving one fractional order of differentiation are not sufficient to describe physical processes. Therefore, recently, several authors have studied more general types of fractional-order stochastic models, such as multi-term equations to obtain analytical and numerical approximation results. For instance, the authors in [20] have studied Euler-Maruyama scheme for fractional stochastic Langevin multi-term equations and introduced a general form of FSLEs together with strong convergence rate of a numerical mild solution.

Among the many scientific articles on asymptotic behaviour and asymptotic separation of fractional stochastic differential equations, we will mention only a few that motivate this work:

- A few works on asymptotic separation of two distinct solutions to fractional stochastic differential equations which can also be found in [21]. Similar work on an exact asymptotic separation rate of two distinct solutions of doubly singular stochastic Volterra integral equations (SVIEs) with two different initial values was discussed in [22].

- Results on the asymptotic behavior of solutions of fractional differential equations with fractional time derivative of Caputo type are relatively rare in the literature. In [23], Cong et al. studied the asymptotic behavior of solutions of the perturbed linear fractional differential system. Cong et al. [24] proved the theorem of linearized asymptotic stability for fractional differential equations.

- The authors in [25] studied the existence and asymptotic stability at the $p$-th moment of a mild solution for a class of nonlinear fractional neutral stochastic differential equations. The results are obtained with the help of the theory of fractional differential equations, some properties of Mittag-Leffler functions and its asymptotic analysis under the assumption that the corresponding fractional stochastic neutral dynamical system is asymptotically stable. The similar asymptotic stability result at the $p$-th moment of a mild solution of nonlinear impulsive stochastic differential equations was discussed in [26,27].

While there are several papers on applications of deterministic fractional multi-term differential equations (FMTDEs) with constant and variable coefficients (see [28-31]), there are few papers dealing with stochastic Caputo stochastic multi-term differential equations (Caputo SMTDEs) involving non-permutable matrices. The lack of asymptotic separation of the solutions of this class of Caputo SMTDEs motivates us to develop new results on asymptotic analysis. The main contributions of our work are described in detail below:

1. We study asymptotic separation between two distinct mild solutions rather than integral equations. This is a lucky consequence which forms an interesting result in its own right.

2. We consider more general Caputo-FSDEs with non-permutable matrices under the weaker condition $\lambda>\alpha$, which is true even in the special case when $\beta=0$ and $A, B$ are equal to zero matrices, than the condition represented in [21]. With respect to this condition, the asymptotic distance between solutions is greater than $t^{-\alpha-\epsilon}$ as $t \rightarrow \infty$ for any $\epsilon>0$.

3. We obtain a bound for the leading coefficient of the asymptotic separation rate for the two distinct solutions which reveals that our asymptotic results are general.

4. As a consequence, the mean square Lyapunov exponent of an arbitrary non-trivial solution of a bounded linear Caputo fractional stochastic differential equation is always non-negative. 
Hence, the plan of this paper is systematized as follows: Section 2 is an introductory section in which we recall the main definitions, results from fractional calculus, and necessary lemmas from fractional differential equations, and in Section 3 we review the framework for the main results of the theory. Section 4 is devoted to proving global existence and uniqueness and continuity dependence on the initial values of the solutions of Caputo SMTDEs of orders $\alpha \in\left(\frac{1}{2}, 1\right)$ and $\beta \in(0,1)$ with $\alpha>\beta$ involving non-permutable matrices. In Section 5, we investigate new results on the asymptotic behavior of solutions of the Caputo SMTDE by studying an asymptotic separation between two different solutions. In Section 6, we present an example to verify the results proved in Sections 4 and 5. Section 7 is for the conclusion.

\section{Mathematical Preliminaries}

We embark on this section by briefly introducing the essential structure of fractional calculus and fractional differential operators. For the more salient details on these matters, see the textbooks [32-36]. Note that none of the results in this section are new, except Definition 2.3 and 2.4 (they are recently defined in [37,38], respectively) at the end, which we place in this section since the Mittag-Leffler type function will be used later in the paper as a representation of solutions.

Definition 1 ([32,34]). The Riemann-Liouville integral operator of fractional order $\alpha>0$ is defined by

$$
I_{0+}^{\alpha} g(t)=\frac{1}{\Gamma(\alpha)} \int_{0}^{t}(t-r)^{\alpha-1} g(r) \mathrm{d} r, \quad \text { for } \quad t>0 .
$$

The Riemann-Liouville derivative operator of fractional order $\alpha>0$ is defined by

$$
D_{0+}^{\alpha} g(t)=\frac{\mathrm{d}^{n}}{\mathrm{~d} t^{n}}\left(I_{0+}^{n-\alpha} g(t)\right), \text { where } n-1<\alpha \leq n .
$$

Definition 2 ([32]). Suppose that $\alpha>0, t>0$. The Caputo derivative operator of fractional order $\alpha$ is defined by:

$$
{ }^{C} D_{0+}^{\alpha} g(t)=I_{0+}^{n-\alpha}\left(\frac{\mathrm{d}^{n}}{\mathrm{~d} t^{n}} g(t)\right), \text { where } n-1<\alpha \leq n .
$$

In particular, for $\alpha \in(0,1)$

$$
I_{0^{+}}^{\alpha}{ }^{C} D_{0^{+}}^{\alpha} f(t)=f(t)-f(0) .
$$

The Riemann-Liouville fractional derivatives have a singularity at zero. That is why differential equations involving these derivatives require initial conditions of special form lacking clear physical interpretation. These shortcomings do not occur with the regularized fractional derivative in the sense of Caputo [33]. For this reason, we consider our main results in the sense of Caputo fractional derivative.

The Riemann-Liouville fractional integral operator and the Caputo fractional derivative have the following property for $\alpha \geq 0$ [32,34]:

$$
I_{0+}^{\alpha}\left({ }^{C} D_{0+}^{\alpha} g(t)\right)=g(t)-\sum_{k=0}^{n-1} \frac{t^{k} g^{(k)}(0)}{\Gamma(k+1)} .
$$

The relationship between the Riemann-Liouville and Caputo fractional derivatives is as follows:

$$
{ }^{C} D_{0+}^{\alpha} g(t)=D_{0+}^{\alpha} g(t)-\sum_{k=0}^{n-1} \frac{t^{k-\alpha} g^{(k)}(0)}{\Gamma(k-\alpha+1)}, \quad \alpha \geq 0 .
$$


Definition 3 ([37]). We define a new Mittag-Leffler type function $E_{\alpha, \beta, \gamma}^{A, B}(\cdot): \mathbb{R} \rightarrow \mathbb{R}$ generated by nonpermutable matrices $A, B \in \mathbb{R}^{n \times n}$ as follows:

$$
E_{\alpha, \beta, \delta}^{A, B}(t)=\sum_{k=0}^{\infty} \sum_{m=0}^{\infty} \mathcal{Q}_{k, m}^{A, B} \frac{t^{k \alpha+m \beta}}{\Gamma(k \alpha+m \beta+\delta)}, \quad \alpha, \beta>0, \quad \delta \in \mathbb{R},
$$

where $\mathcal{Q}_{k, m}^{A, B} \in \mathbb{R}^{n \times n}, k, m \in \mathbb{N}_{0}:=\mathbb{N} \cup\{0\}$ is given by

$$
\mathcal{Q}_{k, m}^{A, B}=\sum_{l=0}^{k} A^{k-l} B \mathcal{Q}_{l, m-1}^{A, B}, \quad k, m \in \mathbb{N}, \quad \mathcal{Q}_{k, 0}^{A, B}=A^{k}, \quad k \in \mathbb{N}_{0}, \quad \mathcal{Q}_{0, m}^{A, B}=B^{m}, \quad m \in \mathbb{N}_{0} .
$$

An explicit representation of $\mathcal{Q}_{k, m}^{A, B}$ can be found in Table 1 in [37]. In the case of permutable matrices, i.e., $A B=B A$, we have $\mathcal{Q}_{k, m}^{A, B}=\left(\begin{array}{c}k+m \\ m\end{array}\right) A^{k} B^{m}, k, m \in \mathbb{N}_{0}$.

Definition 4 ([38]). We consider the Mittag-Leffler type function involving permutable matrices

$$
t^{\delta-1} E_{\alpha, \beta, \delta}\left(B t^{\alpha}, A t^{\beta}\right)=\sum_{k=0}^{\infty} \sum_{m=0}^{\infty}\left(\begin{array}{c}
k+m \\
m
\end{array}\right) \frac{A^{k} B^{m}}{\Gamma(k \alpha+m \beta+\delta)} t^{m \alpha+k \beta+\delta-1} .
$$

The following results are often used to compute estimations in Sections 4 and 5.

Lemma 1. For all $\omega, t>0$, and $\alpha \in\left(\frac{1}{2}, 1\right)$ the following inequality holds:

$$
\frac{\omega}{\Gamma(2 \alpha-1)} \int_{0}^{t}(t-r)^{2 \alpha-2} E_{2 \alpha-1}\left(\omega r^{2 \alpha-1}\right) \mathrm{d} r \leq E_{2 \alpha-1}\left(\omega t^{2 \alpha-1}\right) .
$$

Proof. Applying the series representation of Mittag-Leffler function and definition of beta function, then by swapping summation and integration, we obtain the desired result.

The following lemma plays a necessary role on proofs of main results in Sections 4 and 5.

Lemma 2 (Hölder's inequality). Suppose that $\alpha_{1}, \alpha_{2}>1$ and $\frac{1}{\alpha_{1}}+\frac{1}{\alpha_{2}}=1$. If $|f(t)|^{\alpha_{1}}$, $|g(t)|^{\alpha_{2}} \in L^{1}(\Omega)$, then $|f(t) g(t)| \in L^{1}(\Omega)$ and

$$
\int_{\Omega}|f(t) g(t)| \mathrm{d} t \leq\left(\int_{\Omega}|f(t)|^{\alpha_{1}} \mathrm{~d} t\right)^{\frac{1}{\alpha_{1}}}\left(\int_{\Omega}|g(t)|^{\alpha_{2}} \mathrm{~d} t\right)^{\frac{1}{\alpha_{2}}}
$$

where $L^{1}(\Omega)$ represents the Banach space of all Lebesgue measurable functions $f: \Omega \rightarrow \mathbb{R}$ with $\int_{\Omega}|f(t)| \mathrm{d} t<\infty$. In particular, when $\alpha_{1}=\alpha_{2}=2$, the Hölder's inequality reduces to the Cauchy-Schwartz inequality

$$
\left(\int_{\Omega}|f(t) g(t)| \mathrm{d} t\right)^{2} \leq \int_{\Omega}|f(t)|^{2} \mathrm{~d} t \int_{\Omega}|g(t)|^{2} \mathrm{~d} t .
$$

Lemma 3 (Jensen's inequality). Let $n \in \mathbb{N}, q>1$ and $x_{i} \in \mathbb{R}_{+}, i=1,2, \ldots, n$. Then, the following inequality holds true:

$$
\left\|\sum_{i=1}^{n} x_{i}\right\|^{q} \leq n^{q-1} \sum_{i=1}^{n}\left\|x_{i}\right\|^{q} .
$$

In particular, we consider the following inequality with $q=2$ within the estimations on this paper:

$$
\left\|\sum_{i=1}^{n} x_{i}\right\|^{2} \leq n \sum_{i=1}^{n}\left\|x_{i}\right\|^{2}
$$




\section{Formulation of Main Problem}

In this section, we resort main assumptions which will be used in throughout Section 4 . We consider a Caputo stochastic multi-term differential equation of order $\alpha \in\left(\frac{1}{2}, 1\right)$ and $\beta \in(0,1)$ with $\alpha>\beta$ involving non-permutable matrices has the following form

$$
\left\{\begin{array}{l}
\left({ }^{C} D_{0^{+}}^{\alpha} X\right)(t)-A\left({ }^{C} D_{0^{+}}^{\beta} X\right)(t)-B X(t)=b(t, X(t))+\sigma(t, X(t)) \frac{\mathrm{d} W(t)}{\mathrm{d} t}, \\
X(0)=\eta .
\end{array}\right.
$$

The coefficients $b, \sigma:[0, T] \times \mathbb{R}^{n} \rightarrow \mathbb{R}^{n}$ are measurable and bounded functions. $A, B \in \mathbb{R}^{n \times n}$ are non-permutable matrices. We introduce the norm of the matrix which are used throughout this paper. For any matrix $A=\left(a_{i j}\right)_{n \times n} \in \mathbb{R}^{n \times n}$, the norm of the matrix $A$, according to the maximum norm on $\mathbb{R}^{n}$ is $\|A\|=\max _{1 \leq i \leq n} \sum_{j=1}^{n}\left|a_{i j}\right|$. Moreover, let $\left(W_{t}\right)_{t \geq 0}$ denote a standard scalar Brownian motion on a complete probability space $(\Omega, F, \mathbb{F}, \mathbb{P})$ with filtration $\mathbb{F}=\left\{F_{t}\right\}_{t \in[0, T]}$. The initial condition $\eta$ is an $F_{0}$-measurable $H$-random variable. For each $t \in[0, T]$, let $\Xi_{T}=\mathbb{L}^{2}\left(\Omega, F_{T}, \mathbb{P}\right)$ denote the space of all $F_{T}$ measurable, mean square integrable functions $f=\left(f_{1}, f_{2}, \ldots, f_{n}\right)^{t}: \Omega \rightarrow \mathbb{R}^{n}$ with

$$
\|f\|_{\mathrm{ms}}^{2}=\mathbf{E}\left(\|f\|^{2}\right) \text {. }
$$

Let $H^{2}\left([0, T], \mathbb{R}^{n}\right)$ be well-endowed with the weighted maximum norm $\|\cdot\|_{\omega}$ as

$$
\|\xi\|_{\omega}^{2}=\sup _{t \in[0, T]} \frac{\mathbf{E}\|\xi(t)\|^{2}}{E_{2 \alpha-1}\left(\omega t^{2 \alpha-1}\right)}, \quad \text { for all } \quad \xi \in H^{2}\left([0, T], \mathbb{R}^{n}\right), \omega>0 .
$$

Let $\mathbb{R}^{n}$ be endowed with the standard Euclidean norm and $H^{2}\left([0, T], \mathbb{R}^{n}\right)$ denote the space of all $\mathbb{F}_{T}$-measurable processes $\xi$ satisfying

$$
\|\xi\|_{H^{2}}^{2}=\sup _{t \in[0, T]} \mathbf{E}\|\xi(t)\|^{2}<\infty .
$$

Obviously, $\left(H^{2}\left([0, T], \mathbb{R}^{n}\right),\|\cdot\|_{H^{2}}\right)$ is a Banach space. Since two norms $\|\cdot\|_{H^{2}}$ and $\| \cdot$ $\|_{\omega}$ are equivalent, $\left(H^{2}\left([0, T], \mathbb{R}^{n}\right),\|\cdot\|_{\omega}\right)$ is also Banach space. Therefore, the set $H_{\eta}^{2}\left([0, T], \mathbb{R}^{n}\right)$ is complete with respect to the norm $\|\cdot\|_{\omega}$ and $b, \sigma:[0, T] \times \mathbb{R}^{n} \rightarrow \mathbb{R}^{n}$ are measurable and bounded functions satisfying the following conditions:

Assumption 1. The drift $b$ and diffusion $\sigma$ terms satisfy global Lipschitz continuity: there exists $L_{b}, L_{\sigma}>0$ such that for all $x, y \in \mathbb{R}^{n}, t \in[0, T]$,

$$
\|b(t, x)-b(t, y)\| \leq L_{b}\|x-y\|, \quad\|\sigma(t, x)-\sigma(t, y)\| \leq L_{\sigma}\|x-y\| .
$$

Assumption 2. $b(\cdot, 0)$ is $\mathbb{L}^{2}$ integrable, i.e.

$$
\int_{0}^{T}\|b(r, 0)\|^{2} \mathrm{~d} r<\infty,
$$

and $\sigma(\cdot, 0)$ is essentially bounded, i.e.

$$
\text { ess } \sup _{r \in[0, T]}\|\sigma(r, 0)\|<\infty .
$$

Definition 5. A stochastic process $\{X(t), t \in[0, T]\}$ is called a mild solution of Equation (10) if

- $X(t)$ is adapted to $\left\{F_{t}\right\}_{t \geq 0}$ with $\int_{0}^{t}\|X(t)\|_{H^{2}}^{2} \mathrm{~d} t<\infty$ almost everywhere;

- $X(t) \in H^{2}\left([0, T], \mathbb{R}^{n}\right)$ has continuous path on $t \in[0, T]$ a.s. and satisfies Volterra integral equation of second kind on $t \in[0, T]$ : 


$$
\begin{aligned}
X(t)=\eta-\frac{A t^{\alpha-\beta}}{\Gamma(\alpha-\beta+1)} \eta & +\frac{A}{\Gamma(\alpha-\beta)} \int_{0}^{t}(t-r)^{\alpha-\beta-1} X(r) \mathrm{d} r \\
& +\frac{B}{\Gamma(\alpha)} \int_{0}^{t}(t-r)^{\alpha-1} X(r) \mathrm{d} r \\
& +\frac{1}{\Gamma(\alpha)} \int_{0}^{t}(t-r)^{\alpha-1} b(r, X(r)) \mathrm{d} r \\
& +\frac{1}{\Gamma(\alpha)} \int_{0}^{t}(t-r)^{\alpha-1} \sigma(r, X(r)) \mathrm{d} W(r) .
\end{aligned}
$$

To define above integral equation, we apply Riemann-Liouville integral operator $I_{0+}^{\alpha}$ to the both side of Equation (10), we define

$$
I_{0+}^{\alpha}{ }^{C} D_{0+}^{\alpha} X(t)-A I_{0+}^{\alpha}{ }^{C} D_{0+}^{\beta} X(t)-B I_{0+}^{\alpha} X(t)=I_{0+}^{\alpha} b(t, X(t))+I_{0+}^{\alpha} \sigma(t, X(t)) \frac{\mathrm{d} W(t)}{\mathrm{d} t}
$$

Then we use the relationship between Riemann-Liouville integral and Caputo fractional differential operators Equation (3) for $\frac{1}{2}<\alpha \leq 1$, and $0<\beta \leq 1$, we obtain

$$
\begin{aligned}
X(t) & =\eta+\frac{A}{\Gamma(\alpha)} \int_{0}^{t}(t-r)^{\alpha-1} C^{C} D_{0+}^{\beta} X(r) \mathrm{d} r+\frac{B}{\Gamma(\alpha)} \int_{0}^{t}(t-r)^{\alpha-1} X(r) \mathrm{d} r \\
& +\frac{1}{\Gamma(\alpha)} \int_{0}^{t}(t-r)^{\alpha-1} b(r, X(r)) \mathrm{d} r+\frac{1}{\Gamma(\alpha)} \int_{0}^{t}(t-r)^{\alpha-1} \sigma(r, X(r)) \mathrm{d} W(r),
\end{aligned}
$$

where

$$
\frac{A}{\Gamma(\alpha)} \int_{0}^{t}(t-r)^{\alpha-1 C} D_{0+}^{\beta} X(r) \mathrm{d} r=\frac{A}{\Gamma(\alpha-\beta+1)} \int_{0}^{t}(t-u)^{\alpha-\beta} X^{\prime}(u) \mathrm{d} u .
$$

Then, we apply integration by parts formula for Equation (13) to obtain Equation (12). Now we can represent our mild solution of Equation (10) involving non-permutable matrices.

Lemma 4. Let $A, B \in \mathbb{R}^{n \times n}$ with non-zero commutator, i.e., $[A, B]=A B-B A \neq 0$. For each initial value $\eta \in \Xi_{0}$, the mild solution $X(\cdot) \in \mathbb{R}^{n}$ of the Cauchy problem Equation (10) can be represented in terms of Mittag-Leffler type functions involving non-permutable matrices as below:

$$
\begin{aligned}
X(t) & =\left(I+\sum_{k=0}^{\infty} \sum_{m=0}^{\infty} \mathcal{Q}_{k, m}^{A, B} B \frac{t^{k(\alpha-\beta)+m \alpha+\alpha}}{\Gamma(k(\alpha-\beta)+m \alpha+\alpha+1)}\right) \eta \\
& +\int_{0}^{t} \sum_{k=0}^{\infty} \sum_{m=0}^{\infty} \mathcal{Q}_{k, m}^{A, B} \frac{(t-r)^{k(\alpha-\beta)+m \alpha+\alpha-1}}{\Gamma(k(\alpha-\beta)+m \alpha+\alpha)} b(r, X(r)) \mathrm{d} r \\
& +\int_{0}^{t} \sum_{k=0}^{\infty} \sum_{m=0}^{\infty} \mathcal{Q}_{k, m}^{A, B} \frac{(t-r)^{k(\alpha-\beta)+m \alpha+\alpha-1}}{\Gamma(k(\alpha-\beta)+m \alpha+\alpha)} \sigma(r, X(r)) \mathrm{d} W(r) \\
& =\left(I+t^{\alpha} E_{\alpha-\beta, \alpha, \alpha+1}^{A, B}(t) B\right) \eta+\int_{0}^{t}(t-r)^{\alpha-1} E_{\alpha-\beta, \alpha, \alpha}^{A, B}(t-r) b(r, X(r)) \mathrm{d} r \\
& +\int_{0}^{t}(t-r)^{\alpha-1} E_{\alpha-\beta, \alpha, \alpha}^{A, B}(t-r) \sigma(r, X(r)) \mathrm{d} W(r), \quad t>0 .
\end{aligned}
$$

Lemma 5. As a special case, for each initial value $\eta \in \Xi_{0}$ the system Equation (10) has a unique mild solution in terms of Mittag-Leffler type functions Equation (7) with permutable matrices on $[0, T]$ as below: 


$$
\begin{aligned}
X(t)=\eta & +\eta t^{\alpha} B E_{\alpha, \alpha-\beta, \alpha+1}\left(B t^{\alpha}, A t^{\alpha-\beta}\right) \\
& +\int_{0}^{t}(t-r)^{\alpha-1} E_{\alpha, \alpha-\beta, \alpha}\left(B(t-r)^{\alpha}, A(t-r)^{\alpha-\beta}\right) b(r, X(r)) \mathrm{d} r \\
& +\int_{0}^{t}(t-r)^{\alpha-1} E_{\alpha, \alpha-\beta, \alpha}\left(B(t-r)^{\alpha}, A(t-r)^{\alpha-\beta}\right) \sigma(r, X(r)) \mathrm{d} W(r) .
\end{aligned}
$$

These solutions can be derived with the help of variation of constants formula. Then the coincidence between the notion of mild solution and integral equation of Equation (10) with permutable and non-permutable matrices can be proved in a similar way shown in [11]. Therefore, we omit those proofs here.

\section{Existence and Uniqueness Results and Continuity Dependence on Initial Conditions}

In Section 5, we will look at the behavior of solutions to multi-order systems as the independent variable goes to infinity. For this purpose, it is important to have an existence and uniqueness result. Therefore, our first aim in this research article is to show the global existence and uniqueness of solution of Equation (10). Moreover, we also prove the continuity dependence of solutions on the initial values.

Theorem 1 (Global existence and uniqueness and continuity dependence on the initial values of solutions of Caputo SMTDE). Suppose that Assumptions 1 and 2 hold. Then

(i) for any $\eta \in \Xi_{0}$, the Cauchy problem Equation (10) with initial condition $X(0)=\eta$ has a unique global solution on the whole interval $[0, T]$ denoted by $\varphi(\cdot, \eta)$;

(ii) on any bounded time interval $[0, T]$ with $T>0$, the solution $\varphi(\cdot, \eta)$ depends continuously on $\eta$, i.e.,

$$
\lim _{\eta \rightarrow \gamma} \sup _{t \in[0, T]}\|\varphi(t, \eta)-\varphi(t, \gamma)\|_{m s}^{2}=0 .
$$

For any $\eta \in \Xi_{0}$, we define an operator $\mathcal{T}_{\eta}: H_{\eta}^{2}\left([0, T], \mathbb{R}^{n}\right) \rightarrow H_{\eta}^{2}\left([0, T], \mathbb{R}^{n}\right)$ by

$$
\begin{aligned}
\mathcal{T}_{\eta} Y=\left(I+t^{\alpha} E_{\alpha-\beta, \alpha, \alpha+1}^{A, B}(t) B\right) \eta & +\int_{0}^{t}(t-r)^{\alpha-1} E_{\alpha-\beta, \alpha, \alpha}^{A, B}(t-r) b(r, Y(r)) \mathrm{d} r \\
& +\int_{0}^{t}(t-r)^{\alpha-1} E_{\alpha-\beta, \alpha, \alpha}^{A, B}(t-r) \sigma(r, Y(r)) \mathrm{d} W(r), \quad t>0 .
\end{aligned}
$$

The following lemma is devoted to showing that $\mathcal{T}_{\eta}$ is well-defined.

Lemma 6. For $\eta \in \Xi_{0}$, the operator $\mathcal{T}_{\eta}$ is well-defined.

Proof. Let $Y \in H_{\eta}^{2}\left([0, T], \mathbb{R}^{n}\right)$ be arbitrary. From the definition of $\mathcal{T}_{\eta} Y$ as in Equation (16) and the Jensen's inequality Equation (9) for $n=4$, we have for all $t \in[0, T]$ :

$$
\begin{aligned}
\left\|\left(\mathcal{T}_{\eta} Y\right)(t)\right\|_{\mathrm{ms}}^{2} & \leq 3\|\eta\|_{\mathrm{ms}}^{2}\left\|I+t^{\alpha} E_{\alpha-\beta, \alpha, \alpha+1}^{A, B}(t) B\right\|^{2} \\
& +3 \mathbf{E}\left\|\int_{0}^{t}(t-r)^{\alpha-1} E_{\alpha-\beta, \alpha, \alpha}^{A, B}(t-r) b(r, Y(r)) \mathrm{d} r\right\|^{2} \\
& +3 \mathbf{E}\left\|\int_{0}^{t}(t-r)^{\alpha-1} E_{\alpha-\beta, \alpha, \alpha}^{A, B}(t-r) \sigma(r, Y(r)) \mathrm{d} W(r)\right\|^{2} .
\end{aligned}
$$


Considering $M=\sup _{t \in[0, T]}\left\|E_{\alpha-\beta, \alpha, \alpha}^{A, B}(t)\right\|$ and using Cauchy-Schwarz inequality, we obtain the following results:

$$
\begin{aligned}
\mathbf{E}\left\|\int_{0}^{t}(t-r)^{\alpha-1} E_{\alpha-\beta, \alpha, \alpha}^{A, B}(t-r) b(r, Y(r)) \mathrm{d} r\right\|^{2} & \leq M^{2} \int_{0}^{t}(t-r)^{2 \alpha-2} \mathrm{~d} r \mathbf{E} \int_{0}^{t}\|b(r, Y(r))\|^{2} \mathrm{~d} r \\
& =M^{2} T^{2 \alpha-1} \frac{\mathbf{2}}{2 \alpha-1} \mathbf{E} \int_{0}^{t}\|b(r, Y(r))\|^{2} \mathrm{~d} r .
\end{aligned}
$$

From Assumption 1, we derive

$$
\begin{aligned}
\|b(r, Y(r))\|^{2} & \leq 2\|b(r, Y(r))-b(r, 0)\|^{2}+2\|b(r, 0)\|^{2} \\
& \leq 2 L_{b}^{2}\|Y(r)\|^{2}+2\|b(r, 0)\|^{2} .
\end{aligned}
$$

Therefore, we obtain

$$
\begin{aligned}
\mathbf{E} \int_{0}^{t}\|b(r, Y(r))\|^{2} \mathrm{~d} r & \leq 2 L_{b}^{2} \mathbf{E} \int_{0}^{t}\|Y(r)\|^{2} \mathrm{~d} r+2 \int_{0}^{t}\|b(r, 0)\|^{2} \mathrm{~d} r \\
& \leq 2 L_{b}^{2} T \sup _{r \in[0, T]} \mathbf{E}\|Y(r)\|^{2}+2 \int_{0}^{T}\|b(r, 0)\|^{2} \mathrm{~d} r
\end{aligned}
$$

which together with Equation (18) implies that

$$
\begin{aligned}
\mathbf{E}\left\|\int_{0}^{t}(t-r)^{\alpha-1} E_{\alpha-\beta, \alpha, \alpha}^{A, B}(t-r) b(r, Y(r)) \mathrm{d} r\right\|^{2} & \leq 2 M^{2} \frac{L_{b}^{2} T^{2 \alpha}}{2 \alpha-1}\|Y\|_{H^{2}}^{2} \\
& +2 M^{2} \frac{T^{2 \alpha-1}}{2 \alpha-1} \int_{0}^{T}\|b(r, 0)\|^{2} \mathrm{~d} r .
\end{aligned}
$$

Now using the Itô's isometry, we attain

$$
\begin{aligned}
& \mathbf{E}\left\|\int_{0}^{t}(t-r)^{\alpha-1} E_{\alpha-\beta, \alpha, \alpha}^{A, B}(t-r) \sigma(r, Y(r)) \mathrm{d} W(r)\right\|^{2} \\
& =\sum_{i=1}^{d} \mathbf{E}\left(\int_{0}^{t}(t-r)^{\alpha-1} E_{\alpha-\beta, \alpha, \alpha}^{A, B}(t-r) \sigma_{i}(r, Y(r)) \mathrm{d} W_{r}\right)^{2} \\
& =M^{2} \sum_{i=1}^{d} \mathbf{E}\left(\int_{0}^{t}(t-r)^{2 \alpha-2}\left|\sigma_{i}(r, Y(r))\right|^{2} \mathrm{~d} r\right) \\
& =M^{2} \mathbf{E} \int_{0}^{t}(t-r)^{2 \alpha-2}\|\sigma(r, Y(r))\|^{2} \mathrm{~d} r \\
& \leq M^{2} T^{2 \alpha-2} \mathbf{E} \int_{0}^{t}\|\sigma(r, Y(r))\|^{2} \mathrm{~d} r .
\end{aligned}
$$

From Assumption 1, we also have,

$$
\begin{aligned}
\|\sigma(r, Y(r))\|^{2} & \leq 2\|\sigma(r, Y(r))-\sigma(r, 0)\|^{2}+2\|\sigma(r, 0)\|^{2} \\
& \leq 2 L_{\sigma}^{2}\|Y(r)\|^{2}+2\|\sigma(r, 0)\|^{2} .
\end{aligned}
$$

Therefore, for all $t \in[0, T]$, we have

$$
\begin{aligned}
\mathbf{E}\left\|\int_{0}^{t}(t-r)^{\alpha-1} E_{\alpha-\beta, \alpha, \alpha}^{A, B}(t-r) \sigma(r, Y(r)) \mathrm{d} W(r)\right\|^{2} & \leq 2 M^{2} T^{2 \alpha-2} L_{\sigma}^{2} \mathbf{E} \int_{0}^{t}\|Y(r)\|^{2} \mathrm{~d} r \\
& +2 M^{2} T^{2 \alpha-2} \int_{0}^{t}\|\sigma(r, 0)\|^{2} \mathrm{~d} r \\
& \leq 2 M^{2} L_{\sigma}^{2} T^{2 \alpha-1}\|Y(r)\|_{H^{2}}^{2} \\
& +2 M^{2} T^{2 \alpha-2} \int_{0}^{T}\|\sigma(r, 0)\|^{2} \mathrm{~d} r .
\end{aligned}
$$


This together with Equations (17)-(19) yields that $\left\|\mathcal{T}_{\eta} Y\right\|_{H^{2}}^{2}<\infty$. Hence, the map $\mathcal{T}_{\eta}$ is well-defined.

To prove global existence and uniqueness of solutions, we will show that the operator $\mathcal{T}_{\eta}$ is contractive with respect to the weighted maximum norm Equation (11). Now, we are in a position to prove Theorem 1.

Proof of Theorem 1. Let $T>0$ be an arbitrary. Choose and fix a positive constant $\omega$ such that

$$
\omega>3 \Gamma(2 \alpha-1) M^{2}\left(L_{b}^{2} T+L_{\sigma}^{2}\right) .
$$

(i) Choose and fix $\eta \in \Xi_{0}$. By virtue of Lemma 6 , the operator $\mathcal{T}_{\eta}$ is well-defined. We will prove that the map $\mathcal{T}_{\eta}$ is a contraction with respect to the norm $\|\cdot\|_{\omega}$.

For this purpose, let $X, Y \in H^{2}\left([0, T], \mathbb{R}^{n}\right)$ be arbitrary. From Equation (16) and the inequality Equation (9) with $n=3$, we derive the following estimations for all $t \in[0, T]$ :

$$
\begin{aligned}
\mathbf{E}\left\|\left(\mathcal{T}_{\eta} X\right)(t)-\left(\mathcal{T}_{\eta} Y\right)(t)\right\|^{2} & \leq 2 \mathbf{E}\left\|\int_{0}^{t}(t-r)^{\alpha-1} E_{\alpha-\beta, \alpha, \alpha}^{A, B}(t-r)(b(r, X(r))-b(r, Y(r))) \mathrm{d} r\right\|^{2} \\
& +2 \mathbf{E}\left\|\int_{0}^{t}(t-r)^{\alpha-1} E_{\alpha-\beta, \alpha, \alpha}^{A, B}(t-r)(\sigma(r, X(r))-\sigma(r, Y(r))) \mathrm{d} W(r)\right\|^{2} .
\end{aligned}
$$

Using Cauchy-Schwarz's inequality and Assumption 1, we obtain

$$
\begin{aligned}
& \mathbf{E}\left\|\int_{0}^{t}(t-r)^{\alpha-1} E_{\alpha-\beta, \alpha, \alpha}^{A, B}(t-r)(b(r, X(r))-b(r, Y(r))) \mathrm{d} r\right\|^{2} \\
& \leq M^{2} L_{b}^{2} T \int_{0}^{t}(t-r)^{2 \alpha-2} \mathbf{E}\|X(r)-Y(r)\|^{2} \mathrm{~d} r .
\end{aligned}
$$

Moreover, by Itô's isometry and Assumption 1, we also have

$$
\begin{aligned}
& \mathbf{E}\left\|\int_{0}^{t}(t-r)^{\alpha-1} E_{\alpha-\beta, \alpha, \alpha}^{A, B}(t-r)(\sigma(r, X(r))-\sigma(r, Y(r))) \mathrm{d} W(r)\right\|^{2} \\
& \leq M^{2} L_{\sigma}^{2} \int_{0}^{t}(t-r)^{2 \alpha-2} \mathbf{E}\|X(r)-Y(r)\|^{2} \mathrm{~d} r .
\end{aligned}
$$

Then for all $t \in[0, T]$, we acquire

$$
\mathbf{E}\left\|\left(\mathcal{T}_{\eta} X\right)(t)-\left(\mathcal{T}_{\eta} Y\right)(t)\right\|^{2} \leq 2 M^{2}\left(L_{b}^{2} T+L_{\sigma}^{2}\right) \int_{0}^{t}(t-r)^{2 \alpha-2} \mathbf{E}\|X(r)-Y(r)\|^{2} \mathrm{~d} r,
$$

which together with the definition of $\|\cdot\|_{\omega}$ as in Equation (11) implies that

$$
\begin{aligned}
\frac{\mathbf{E}\left\|\left(\mathcal{T}_{\eta} X\right)(t)-\left(\mathcal{T}_{\eta} Y\right)(t)\right\|^{2}}{E_{2 \alpha-1}\left(\omega t^{2 \alpha-1}\right)} & \leq 2 M^{2}\left(L_{b}^{2} T+L_{\sigma}^{2}\right) \frac{1}{E_{2 \alpha-1}\left(\omega t^{2 \alpha-1}\right)} \\
& \times \int_{0}^{t}(t-r)^{2 \alpha-2} E_{2 \alpha-1}\left(\omega r^{2 \alpha-1}\right) \frac{\mathbf{E}\|X(r)-Y(r)\|^{2}}{E_{2 \alpha-1}\left(\omega r^{2 \alpha-1}\right)} \mathrm{d} r .
\end{aligned}
$$

By virtue of Lemma 1, we have for all $t \in[0, T]$ :

$$
\frac{\mathbf{E}\left\|\left(\mathcal{T}_{\eta} X\right)(t)-\left(\mathcal{T}_{\eta} Y\right)(t)\right\|^{2}}{E_{2 \alpha-1}\left(\omega t^{2 \alpha-1}\right)} \leq \frac{2 \Gamma(2 \alpha-1)}{\omega} M^{2}\left(L_{b}^{2} T+L_{\sigma}^{2}\right)\|X-Y\|_{\omega}^{2} .
$$


As a consequence,

$$
\left\|\mathcal{T}_{\eta} X-\mathcal{T}_{\eta} Y\right\|_{\omega}^{2} \leq \zeta\|X-Y\|_{\omega}^{2}
$$

where

$$
\zeta=\frac{2 \Gamma(2 \alpha-1)}{\omega} M^{2}\left(L_{b}^{2} T+L_{\sigma}^{2}\right)
$$

By Equation (20), we have $\zeta<1$ and the operator $\mathcal{T}_{\eta}$ is a contractive mapping on $H^{2}\left([0, T],\|\cdot\|_{\omega}\right)$. Using the Banach's fixed point theorem, there exists a unique fixed point of this map in $H^{2}\left([0, T], \mathbb{R}^{n}\right)$. This fixed point is also a unique solution of Equation (10) with initial conditions $X(0)=\eta$. The proof of (i) is complete.

(ii) Choose and fix $T>0$ and $\eta, \gamma \in \Xi_{0}$. Since $\varphi(\cdot, \eta)$ and $\varphi(\cdot, \gamma)$ are solution of Equation (10), it follows that

$$
\begin{aligned}
\varphi(t, \eta)-\varphi(t, \gamma) & =(\eta-\gamma)\left(I+t^{\alpha} E_{\alpha-\beta, \alpha, \alpha+1}^{A, B}(t) B\right) \\
& +\int_{0}^{t}(t-r)^{\alpha-1} E_{\alpha-\beta, \alpha, \alpha}^{A, B}(t-r)(b(r, \varphi(r, \eta))-b(r, \varphi(r, \gamma))) \mathrm{d} r \\
& +\int_{0}^{t}(t-r)^{\alpha-1} E_{\alpha-\beta, \alpha, \alpha}^{A, B}(t-r)(\sigma(r, \varphi(r, \eta))-\sigma(r, \varphi(r, \gamma))) \mathrm{d} W(r) .
\end{aligned}
$$

Hence, using the Jensen's inequality Equation (9) for $n=3$, Assumption 1 and 2, the Cauchy-Schwarz inequality and Itô's isometry, we obtain

$$
\begin{aligned}
\mathbf{E}\|\varphi(t, \eta)-\varphi(t, \gamma)\|^{2} & \leq 3 \mathcal{C} \mathbf{E}\|\eta-\gamma\|^{2} \\
& +3 \frac{\Gamma(2 \alpha-1)}{\omega} M^{2}\left(L_{b}^{2} T+L_{\sigma}^{2}\right) \int_{0}^{t}(t-r)^{2 \alpha-2} \mathbf{E}\|\varphi(r, \eta)-\varphi(r, \gamma)\|^{2} \mathrm{~d} r
\end{aligned}
$$

where $\mathcal{C}:=\left\|I+t^{\alpha} E_{\alpha-\beta, \alpha, \alpha+1}^{A, B}(t) B\right\|^{2}$.

By virtue of Lemma 1 and the definition of $\|\cdot\|_{\omega}$, we have

$$
\frac{\mathbf{E}\|\varphi(t, \eta)-\varphi(t, \gamma)\|^{2}}{E_{2 \alpha-1}\left(\omega t^{2 \alpha-1}\right)} \leq 3 \mathcal{C}\|\eta-\gamma\|_{\mathrm{ms}}^{2}+\zeta\|\varphi(t, \eta)-\varphi(t, \gamma)\|_{\omega}^{2}
$$

Thus, by Equation (20), we have

$$
(1-\zeta)\|\varphi(t, \eta)-\varphi(t, \gamma)\|_{\omega}^{2} \leq 3 \mathcal{C}\|\eta-\gamma\|_{\mathrm{ms}}^{2}
$$

Hence,

$$
\lim _{\eta \rightarrow \gamma} \sup _{t \in[0, T]}\|\varphi(t, \eta)-\varphi(t, \gamma)\|_{\mathrm{ms}}^{2}=0
$$

The proof is complete.

\section{Asymptotic Separation between Mild Solutions of Equation (10)}

Theorem 2. Let $\eta, \gamma \in \Xi_{0}$ such that $\eta \neq \gamma$. Then for any $\epsilon>0$

$$
\limsup _{t \rightarrow \infty} t^{\alpha+\epsilon}\|\varphi(t, \eta)-\varphi(t, \gamma)\|_{m s}=\infty
$$

Proof. Assume the contrary, i.e., there exists a positive constant $\lambda>\alpha$ such that

$$
\limsup _{t \rightarrow \infty} t^{\lambda}\|\varphi(t, \eta)-\varphi(t, \gamma)\|_{\mathrm{ms}}<\infty
$$


for some $\eta, \gamma \in \Xi_{0}, \eta \neq \gamma$. There exists constants $T>0$ and $\kappa>0$ such that

$$
\|\varphi(t, \eta)-\varphi(t, \gamma)\|_{\mathrm{ms}}^{2} \leq \kappa t^{-2 \lambda} \text { for all } t \geq T .
$$

From Equation (12) and the inequality Equation (9), we have

$$
\begin{aligned}
\|\eta-\gamma\|^{2} & \leq \frac{1}{\mathcal{C} \mathcal{C}}\|\varphi(t, \eta)-\varphi(t, \gamma)\|^{2} \\
& +\frac{1}{\mathcal{C}}\left\|\int_{0}^{t}(t-r)^{\alpha-1} E_{\alpha-\beta, \alpha, \alpha}^{A, B}(t-r)(b(r, \varphi(r, \eta))-b(r, \varphi(r, \gamma))) \mathrm{d} r\right\|^{2} \\
& +\frac{1}{\mathcal{C}}\left\|\int_{0}^{t}(t-r)^{\alpha-1} E_{\alpha-\beta, \alpha, \alpha}^{A, B}(t-r)(\sigma(r, \varphi(r, \eta))-\sigma(r, \varphi(r, \gamma))) \mathrm{d} W(r)\right\|^{2} .
\end{aligned}
$$

Taking expectation of both sides and using Assumption 1, we obtain

$$
\begin{aligned}
\|\eta-\gamma\|_{\mathrm{ms}}^{2} & \leq \frac{1}{\mathcal{C}} \mathbf{E}\|\varphi(t, \eta)-\varphi(t, \gamma)\|^{2} \\
& +\frac{1}{\mathcal{C}} \mathbf{E}\left(\int_{0}^{t}(t-r)^{\alpha-1}\left\|E_{\alpha-\beta, \alpha, \alpha}^{A, B}(t-r)\right\| L_{b}\|\varphi(r, \eta)-\varphi(r, \gamma)\| \mathrm{d} r\right)^{2} \\
& +\frac{1}{\mathcal{C}} \mathbf{E}\left(\int_{0}^{t}(t-r)^{\alpha-1}\left\|E_{\alpha-\beta, \alpha, \alpha}^{A, B}(t-r)\right\| L_{\sigma}\|\varphi(r, \eta)-\varphi(r, \gamma)\| \mathrm{d} W(r)\right)^{2} .
\end{aligned}
$$

From Equation (21), we derive that $\lim _{t \rightarrow \infty} \mathbf{E}\|\varphi(r, \eta)-\varphi(r, \gamma)\|^{2}=0$. Thus, to derive contradiction it is enough to show that

$$
\lim _{t \rightarrow \infty} \mathcal{I}_{1}(t)=0, \quad \text { where } \quad \mathcal{I}_{1}(t)=\mathbf{E}\left(\int_{0}^{t}(t-r)^{\alpha-1}\left\|E_{\alpha-\beta, \alpha, \alpha}^{A, B}(t-r)\right\| L_{b}\|\varphi(r, \eta)-\varphi(r, \gamma)\| \mathrm{d} r\right)^{2}
$$

and

$$
\lim _{t \rightarrow \infty} \mathcal{I}_{2}(t)=0, \quad \text { where } \quad \mathcal{I}_{2}(t)=M^{2} L_{\sigma}^{2} \int_{0}^{t}(t-r)^{2 \alpha-2}\|\varphi(r, \eta)-\varphi(r, \gamma)\|_{\mathrm{ms}}^{2} \mathrm{~d} r
$$

To show Equation (22), choose and fix $\delta \in\left(\frac{\alpha}{\lambda}, 1-\alpha\right)$. Please note that the existence of such a $\delta$ comes from the fact that $\frac{\alpha}{\lambda}<1-\alpha$ that is equivalent to the assumption $\lambda>\frac{\alpha}{1-\alpha}$. For $t>\max \left\{T^{1 / \delta}, 1\right\}$, using Cauchy-Schwarz's inequality and the inequality Equation (21), we have

$$
\begin{aligned}
\mathcal{I}_{1}(t) & \leq 2 \mathbf{E}\left(\int_{0}^{t^{\delta}}(t-r)^{\alpha-1}\left\|E_{\alpha-\beta, \alpha, \alpha}^{A, B}(t-r)\right\| L_{b}\|\varphi(r, \eta)-\varphi(r, \gamma)\| \mathrm{d} r\right)^{2}, \\
& +2 \mathbf{E}\left(\int_{t^{\delta}}^{t}(t-r)^{\alpha-1}\left\|E_{\alpha-\beta, \alpha, \alpha}^{A, B}(t-r)\right\| L_{b}\|\varphi(r, \eta)-\varphi(r, \gamma)\| \mathrm{d} r\right)^{2}, \\
& \leq 2 M^{2} L_{b}^{2} \int_{0}^{t^{\delta}}(t-r)^{2 \alpha-2} \mathrm{~d} r \int_{0}^{t^{\delta}}\|\varphi(r, \eta)-\varphi(r, \gamma)\|_{\mathrm{ms}}^{2} \mathrm{~d} r \\
& +2 M^{2} L_{b}^{2} \int_{t^{\delta}}^{t}(t-r)^{2 \alpha-2} \mathrm{~d} r \int_{t^{\delta}}^{t}\|\varphi(r, \eta)-\varphi(r, \gamma)\|_{\mathrm{ms}}^{2} \mathrm{~d} r .
\end{aligned}
$$


Since

$$
\int_{0}^{t^{\delta}}(t-r)^{2 \alpha-2} \mathrm{~d} r=\frac{t^{\delta}}{\left(t-t^{\delta}\right)^{2-2 \alpha}}, \quad \int_{t^{\delta}}^{t}(t-r)^{2 \alpha-2} \mathrm{~d} r=\frac{\left(t-t^{\delta}\right)^{2 \alpha-1}}{2 \alpha-1},
$$

it yields together with Equation (21) that

$$
\begin{aligned}
\mathcal{I}_{1}(t) & \leq \frac{2 M^{2} L_{b}^{2} t^{2 \delta}}{\left(t-t^{\delta}\right)^{2-2 \alpha}} \sup _{r \in[0, T]}\|\varphi(r, \eta)-\varphi(r, \gamma)\|_{\mathrm{ms}}^{2}+2 \kappa M^{2} L_{b}^{2} \frac{\left(t-t^{\delta}\right)^{2 \alpha-1}}{2 \alpha-1} \int_{t^{\delta}}^{t} r^{-2 \lambda} \mathrm{d} r \\
& \leq \frac{2 M^{2} L_{b}^{2} t^{2 \delta}}{\left(t-t^{\delta}\right)^{2-2 \alpha}} \sup _{r \in[0, T]}\|\varphi(r, \eta)-\varphi(r, \gamma)\|_{\mathrm{ms}}^{2}+\frac{2 M^{2} L_{b}^{2} \kappa\left(t-t^{\delta}\right)^{2 \alpha}}{(2 \alpha-1) t^{2 \delta \lambda}}
\end{aligned}
$$

By definition of $\delta$, we have $2 \delta<2-2 \alpha$ and $2 \alpha<2 \delta \lambda$. Hence, letting $t \rightarrow \infty$ in the expression Equation (24) yields $\lim _{t \rightarrow \infty} I_{1}(t)=0$. Therefore, Equation (22) is proved. we have

Concerning the assertion Equation (23), let $t \geq T$ be arbitrary. By Equation (21),

$$
\begin{aligned}
\mathcal{I}_{2}(t) & \leq M^{2} L_{\sigma}^{2} \int_{0}^{T}(t-r)^{2 \alpha-2}\|\varphi(r, \eta)-\varphi(r, \gamma)\|_{\mathrm{ms}}^{2} \mathrm{~d} r+\kappa M^{2} L_{\sigma}^{2} \int_{T}^{t}(t-r)^{2 \alpha-2} r^{-2 \lambda} \mathrm{d} r \\
& \leq M^{2} L_{\sigma}^{2} \frac{T}{(t-T)^{2-2 \alpha}} \sup _{r \in[0, T]}\|\varphi(r, \eta)-\varphi(r, \gamma)\|_{\mathrm{ms}}^{2}+\kappa M^{2} L_{\sigma}^{2} \int_{T}^{t}(t-r)^{2 \alpha-2} r^{-2 \lambda} \mathrm{d} r .
\end{aligned}
$$

Therefore,

$$
\limsup _{t \rightarrow \infty} \mathcal{I}_{2}(t) \leq \kappa M^{2} L_{\sigma}^{2} \limsup _{t \rightarrow \infty} \int_{T}^{t}(t-r)^{2 \alpha-2} r^{-2 \lambda} \mathrm{d} r .
$$

Please note that for $t \geq 2 T$, we have

$$
\begin{aligned}
\int_{T}^{t}(t-r)^{2 \alpha-2} r^{-2 \lambda} \mathrm{d} r & =\int_{T}^{t / 2}(t-r)^{2 \alpha-2} r^{-2 \lambda} \mathrm{d} r+\int_{t / 2}^{t}(t-r)^{2 \alpha-2} r^{-2 \lambda} \mathrm{d} r \\
& \leq \frac{2^{2-2 \alpha}}{t^{2-2 \alpha}} \int_{T}^{t / 2} r^{-2 \lambda} \mathrm{d} r+\left(\frac{t}{2}\right)^{-2 \lambda} \int_{t / 2}^{t}(t-r)^{2 \alpha-2} \mathrm{~d} r \\
& \leq \frac{2^{2-2 \alpha} T^{-2 \lambda+1}}{(2 \lambda-1) t^{2-2 \alpha}}+\frac{1}{2 \alpha-1}\left(\frac{t}{2}\right)^{2 \alpha-2 \lambda-1},
\end{aligned}
$$

which together with Equation (25) and the fact that $\alpha \in\left(\frac{1}{2}, 1\right)$ and $\lambda>\frac{\alpha}{1-\alpha}>\alpha$, implies that $\lim _{t \rightarrow \infty} \mathcal{I}_{2}(t)=0$. Thus, Equation (23) is proved and therefore the proof is complete.

As a consequence, we give an application of the main results concerning the mean square Lyapunov exponent on non-trivial solutions to a bounded bilinear Caputo FSDE. To formulate this result, we consider the following equation:

$$
\left\{\begin{array}{l}
\left({ }^{C} D_{0^{+}}^{\alpha} X\right)(t)-\lambda\left({ }^{C} D_{0^{+}}^{\beta} X\right)(t)-\mu X(t)=A(t) X(t)+B(t) X(t) \frac{\mathrm{d} W(t)}{\mathrm{d} t}, \\
X(0)=\eta
\end{array}\right.
$$

where $A(\cdot), B(\cdot) \in[0, \infty) \rightarrow \mathbb{R}^{n \times n}$ are measurable and essentially bounded, i.e.,

$$
t \in[0, \infty)\|A(t)\|<\infty, \quad t \in[0, \infty)\|B(t)\|<\infty .
$$

By virtue of Theorem 1, for any $\eta \in \Xi_{0} \backslash\{0\}$, there exists a unique solution of Equation (26), denoted by $\Psi(\cdot, \eta)$ satisfying initial condition. The mean square Lyapunov exponent of $\Psi(\cdot, \eta)$ is defined by (see $[39,40]$ )

$$
\Lambda_{\mathrm{ms}}(\Psi(\cdot, \eta)):=\limsup _{t \rightarrow \infty} \frac{1}{t} \ln \|\Psi(\cdot, \eta)\|_{\mathrm{ms}}
$$


In the following corollary, we show the non-negativity of the mean square Lyapunov exponent of an arbitrary non-trivial solution.

Corollary 1. The mean square Lyapunov exponent of a nontrivial solution of Equation (26) is always non-negative, i.e.,

$$
\Lambda_{m s}(\Psi(\cdot, \eta)) \geq 0, \quad \forall \eta \in \Xi_{0} \backslash\{0\} .
$$

Proof. Let $\eta \in \Xi_{0} \backslash\{0\}$ be arbitrary. Using Theorem 2, we attain

$$
\limsup _{t \rightarrow \infty} t^{\alpha+\epsilon}\|\Psi(t, \eta)\|_{\mathrm{ms}}=\infty, \quad \epsilon>0 .
$$

Hence, there exists $T>0$ such that

$$
\|\Psi(t, \eta)\|_{\mathrm{ms}} \geq t^{-\alpha-\epsilon}, \quad \forall t \geq T,
$$

which together with Equation (27) implies that

$$
\Lambda_{\mathrm{ms}}(\Psi(\cdot, \eta)) \geq \limsup _{t \rightarrow \infty} \frac{1}{t} \ln \left(t^{-(\alpha+\epsilon)}\right)=0 .
$$

Remark 1. In special case, $\beta=0$ and $A, B=\Theta$ are zero matrices, the system Equation (10) can be reduced to the following Caputo fractional stochastic differential Equation (Caputo FSDE):

$$
\left\{\begin{array}{l}
\left({ }^{C} D_{0^{+}}^{\alpha} X\right)(t)=b(t, X(t))+\sigma(t, X(t)) \frac{\mathrm{d} W(t)}{\mathrm{d} t}, \\
X(0)=\eta
\end{array}\right.
$$

in which asymptotic separation between solutions of above Caputo FSDE has been discussed with the help of Theorem 2 in [21]. The main point in [21] is that $\lambda$ is chosen as $\lambda>\frac{2 \alpha}{1-\alpha}$. We study asymptotic separation between mild solutions rather than integral equations. Moreover, unlike Cong et al. [21], we consider more general Caputo FSDEs with non-permutable matrices under weaker condition $\lambda>\alpha$ than [21]. With regards to this condition, the asymptotic separation between the solutions is greater than $t^{-\alpha-\epsilon}$ as $t \rightarrow \infty$ for any $\epsilon>0$.

Remark 2. It is worthy pointing out that some changes can be made to extend the results for Riemann-Liouville type SMTDEs. Therefore, we consider the following form of RL type SMTDEs of fractional orders $\alpha \in\left(\frac{1}{2}, 1\right)$ and $\beta \in(0,1)$ involving non-permutable matrices:

$$
\left\{\begin{array}{l}
\left(D_{0^{+}}^{\alpha} X\right)(t)-A\left(D_{0^{+}}^{\beta} X\right)(t)-B X(t)=b(t, X(t))+\sigma(t, X(t)) \frac{\mathrm{d} W(t)}{\mathrm{d} t} \\
\left.I_{0^{+}}^{1-\alpha} X(t)\right|_{t=0}=\eta
\end{array}\right.
$$

Then the solution of Equation (29) will become as follows:

$$
\begin{aligned}
X(t) & =t^{\alpha-1} E_{\alpha-\beta, \alpha, \alpha}^{A, B}(t) \eta+\int_{0}^{t}(t-r)^{\alpha-1} E_{\alpha-\beta, \alpha, \alpha}^{A, B}(t-r) b(r, X(r)) \mathrm{d} r \\
& +\int_{0}^{t}(t-r)^{\alpha-1} E_{\alpha-\beta, \alpha, \alpha}^{A, B}(t-r) \sigma(r, X(r)) \mathrm{d} W(r), \quad t>0 .
\end{aligned}
$$


Analogously, to show existence and uniqueness results and continuity dependence on initial conditions we consider the following corresponding weighted maximum norm instead of Equation (11) in order to avoid singularity:

$$
\|\xi\|_{\omega}^{2}=\sup _{t \in[0, T]} \frac{t^{1-\alpha} \mathbf{E}\|\xi(t)\|^{2}}{E_{2 \alpha-1}\left(\omega t^{2 \alpha-1}\right)}, \quad \text { for all } \quad \xi \in H^{2}\left([0, T], \mathbb{R}^{n}\right), \omega>0 .
$$

Therefore, the asymptotic separation property between two distinct solutions of Equation (29) agrees with the same condition, i.e., $\lambda>\alpha$ as stated in Theorem 2.

\section{Example}

Now we provide examples to support the theory developed in the previous sections. We consider the nonlinear fractional stochastic equation system with $\alpha=\frac{3}{4}$ and $\beta=\frac{1}{4}$

$$
\left\{\begin{array}{l}
\left({ }^{C} D_{0^{+}}^{3 / 4} X\right)(t)-\left(\begin{array}{ll}
0.1 & 0.2 \\
0.3 & 0.4
\end{array}\right)\left({ }^{C} D_{0^{+}}^{1 / 4} X\right)(t)-\left(\begin{array}{ll}
0.4 & 0.1 \\
0.2 & 0.3
\end{array}\right) X(t) \\
=\left[\begin{array}{l}
\sin X_{1} \\
X_{2}+5
\end{array}\right]+\left[\begin{array}{l}
X_{1}+5 \\
\cos X_{2}
\end{array}\right] \frac{\mathrm{d} W(t)}{\mathrm{d} t}, \quad t \in[0,1] \\
X(0)=\left[\begin{array}{l}
3 \\
5
\end{array}\right]
\end{array}\right.
$$

where $X(t)=\left(X_{1}(t), X_{2}(t)\right)^{T}$ and $W(t)$ is a Wiener process. By comparison with Equation (10) we have

$$
\begin{aligned}
& A=\left(\begin{array}{ll}
0.1 & 0.2 \\
0.3 & 0.4
\end{array}\right), B=\left(\begin{array}{ll}
0.4 & 0.1 \\
0.2 & 0.3
\end{array}\right), \\
& b(t, X(t))=\left[\begin{array}{l}
\sin X_{1} \\
X_{2}+5
\end{array}\right], \quad \sigma(t, X(t))=\left[\begin{array}{l}
X_{1}+5 \\
\cos X_{2}
\end{array}\right] .
\end{aligned}
$$

It is obvious that $A B \neq B A$. Then, the unique mild solution of Equation (32) involving non-permutable matrices is given by

$$
\begin{aligned}
X(t)= & \left(I+t^{\frac{3}{4}} E_{\frac{1}{2}, \frac{3, \frac{7}{4}}{4}}^{A, B}(t) B\right) X(0)+\int_{0}^{t}(t-r)^{-\frac{1}{4}} E_{\frac{1}{2}, \frac{3}{4}, \frac{3}{4}}^{A, B}(t-r) b(r, X(r)) \mathrm{d} r \\
& +\int_{0}^{t}(t-r)^{-\frac{1}{4}} E_{\frac{1}{2}, \frac{3}{4}, \frac{3}{4}}^{A, B}(t-r) \sigma(r, X(r)) \mathrm{d} W(r) .
\end{aligned}
$$

It is clear that $b(t, X(t)), \sigma(t, X(t))$ satisfy Assumptions 1 and 2. Then all the hypotheses of Theorem 1 are so verified and hence, Equation (32) has a mild solution on $H_{\eta}^{2}\left([0,1], \mathbb{R}^{n}\right)$. We can then validate asymptotic separation property between two distinct mild solutions of Caputo SMTDEs Equation (32) according to Theorem 2, i.e., $\limsup t^{\frac{3}{4}+\epsilon}\|\varphi(t, \eta)-\varphi(t, \gamma)\|_{\mathrm{ms}}=\infty$ for any $\epsilon>0$ and $\eta \neq \gamma, \eta, \gamma \in \Xi_{0}$.

\section{Conclusions}

In this paper, we studied Caputo SMTDEs with matrix coefficients that are not permutable. The fractional orders of differentiation $\alpha$ and $\beta$ are also assumed to be $\left(\frac{1}{2}, 1\right)$ and $(0,1)$, respectively. However, they are completely independent of each other.

The core of this paper is to study asymptotic separation of two mild solutions involving non-permutable matrices of fractional stochastic multi-term differential equations and to provide a proof of the existence and uniqueness of Equation (10) under some natural assumptions on the coefficients. Moreover, we also determine the asymptotic separation 
between two different solutions of Equation (10), where the asymptotic distance $\infty$ is as $t \rightarrow \infty$ when $\lambda>\alpha$, implying that $\lambda$ does not depend on $\beta$.

As a consequence of the main theorems constructing solution functions to Caputo SMTDE systems, by comparing these results with some existing results in the literature proved from the constant coefficients point of view, we were able to find a more general condition based on $\lambda$ which is valid for a class of fractional stochastic differential equations with a single derivative of fractional order. This is a lucky consequence which forms an interesting result in its own right.

Although the asymptotic separation of two distinct mild solutions have now been constructed, there remain many other interesting open problems to be considered regarding asymptotics of the solution functions which may be studied by methods analogous to those used for computing asymptotics of Mittag-Leffler type functions in the univariate case.

Other related research directions may include the various relevant function spaces that may be useful in the qualitative theory of fractional stochastic differential equations associated with these operators, e.g., well-posedness and regularity theory [41].

Author Contributions: Formal analysis, A.A.; Investigation, A.A.; Methodology, A.A. and N.M.; Supervision, N.M.; Validation, N.M.; Writing—original draft, A.A.; Writing—review \& editing, A.A. and N.M. All authors have read and agreed to the published version of the manuscript.

Funding: The author declares that there is no funding information available.

Institutional Review Board Statement: Not applicable.

Informed Consent Statement: Not applicable.

Data Availability Statement: Not applicable.

Conflicts of Interest: The authors declare no conflict of interest.

\section{References}

1. Itô, K. Stochastic Differential Equations. Mem. Am. Math. Soc. 1951, 4, 1-51.

2. Prato, G.D.; Zabczyk, J. Stochastic Equations in Infinite Dimensions; Cambridge University Press: Cambridge, UK, 1992.

3. Oksendal, B. Stochastic Differential Equations: An Introduction with Applications; Springer: Berlin/Heidelberg, Germany, 2000.

4. Gardiner, C.W. Stochastic Methods: For Physics, Chemistry and the Natural Sciences; Springer: Berlin/Heidelberg, Germany, 2004.

5. Mikosch, T. Elementary Stochastic Calculus: With Finance in View; World Scientific Publishing: Singapore, 1998.

6. Tien, D.N. Fractional stochastic differential equations with applications to finance. J. Math. Anal. Appl. 2013, 397, 334-348. [CrossRef]

7. Farhadi, A.; Erjaee, G.H.; Salehi, M. Derivation of a new Merton's optimal problem presented by fractional stochastic stock price and its applications. Comput. Math. Appl. 2017, 73, 2066-2075. [CrossRef]

8. Wang, Y.; Zheng, S.; Zhang, W.; Wang, J. Complex and entropy of fluctuations of agent-based interacting financial dynamics with random jump. Entropy 2017, 19, 512. [CrossRef]

9. Jean, P.C.; Gangaram, L.S. Stochastic fractional differential equations: Modeling, method and analysis. Chaos Solitons Fract. 2012, 45, 279-293.

10. Mandelbrot, B.; Ness, J.V. Fractional Brownian motions, fractional noises and applications. SIAM Rev. 1968, 10, 422-437. [CrossRef]

11. Ahmadova, A.; Mahmudov, N.I. Existence and uniqueness results for a class of fractional stochastic neutral differential equations. Chaos Solitons Fract. 2020, 139, 110253. [CrossRef]

12. Rodkina, A.E. On existence and uniqueness of solution of stochastic differential equations with heredity. Stoch. Monogr. 1984, 12, 187-200. [CrossRef]

13. Taniguchi, T.; Liu, K.; Truman, A. Existence and uniqueness and asymptotic behavior of mild solutions to stochastic functional differential equations in Hilbert spaces. Differ. Equ. 2002, 181, 72-91. [CrossRef]

14. Barbu, D. Local and global existence for mild solutions of stochastic differential equations. Port. Math. 1998, 55, 411-424.

15. Sakthivel, R.; Luo, J. Asymptotic stability of impulsive stochastic partial differential equations with infinite delays. J. Math. Anal. Appl. 2009, 356, 1-6. [CrossRef]

16. Shen, G.; Sakthivel, R.; Ren, Y.; Li, M. Controllability and stability of fractional stochastic functional systems driven by Rosenblatt process. Collect. Math. 2020, 71, 63-82; doi: 10.1007/s13348-019-00248-3. [CrossRef]

17. Mao, X. Exponential Stability of Stochastic Differential Equations; Marcel Dekker: New York, NY, USA, 1994.

18. Beck, C.; Roepstorff, G. From dynamical systems to the langevin equation. Phys. A Stat. Mech. Appl. 1987, 145, 1-14. [CrossRef]

19. WCoffey, T.; Kalmykov, Y.P.; Waldron, J.T. The Langevin Equation, 2nd ed.; World Scientific: Singapore, 2004. 
20. Ahmadova, A.; Mahmudov, N.I. Strong convergence of a Euler-Maruyama method for fractional stochastic Langevin equations. Math. Comput. Simul. 2021, 190, 429-448. [CrossRef]

21. Son, D.T.; Huong, P.T.; Kloeden, P.E.; Tuan, H.T. Asymptotic separation between solutions of Caputo fractional stochastic differential equations. Stoc. Anal. Appl. 2018, 36, 654-664. [CrossRef]

22. Li, M.; Huang, C.; Hu, Y. Asymptotic separation for stochastic Volterra integral equations with doubly singular kernels. Appl. Math. Lett. 2021, 113, 106880. [CrossRef]

23. Cong, N.D.; Doan, T.S.; Tuan, H.T. Asymptotic Stability of Linear Fractional Systems with Constant Coefficients and Small Time-Dependent Perturbations. Vietnam J. Math. 2018, 46, 665-680. [CrossRef]

24. Cong, N.D.; Doan, T.S.; Sigmund, S.; Tuan, H.T. Linearized asymptotic stability for fractional differential equations. Electron. J. Qual. Theory Differ. Equ. 2016, 39, 1-13. [CrossRef]

25. Ahmadova, A.; Mahmudov, N.I. Asymptotic stability analysis of Riemann-Liouville fractional stochastic neutral differential equations. arXiv 2021, arXiv:2109.11493.

26. Sakthivel, R.; Revathi, P.; Mahmudov, N.I. Asymptotic stability of fractional stochastic neutral differential equations with infinite delays. Abstr. Appl. Anal. 2012, 51, 1-9. [CrossRef]

27. Sakthivel, R.; Luo, J. Asymptotic stability of nonlinear impulsive stochastic differential equations. Stat Probab Lett. 2009, 79, 1219-1223. [CrossRef]

28. Ahmadova, A.; Huseynov, I.T.; Fernandez, A.; Mahmudov, N.I. Trivariate Mittag-Leffler functions used to solve multi-order systems of fractional differential equations. Commun. Nonlinear Sci. Numer. Simulat. 2021, 97, 105735. [CrossRef]

29. Fernandez, A.; Restrepo, J.E.; Suragan, D. A new representation for the solutions of fractional differential equations with variable coefficients. arXiv 2021, arXiv:2105.00870.

30. Patnaik, S.; Hollkamp, J.P.; Semperlotti, F. Applications of variable-order fractional operators: A review. Proc. R. Soc. A 2020, 476, 2234. [CrossRef] [PubMed]

31. Rahimi, Z.; Sumelka, W.; Yang, X.J. A new fractional nonlocal model and its application in free vibration of Timoshenko and Euler-Bernoulli beams. Eur. Phys. J. Plus 2017, 132, 479. [CrossRef]

32. Podlubny, I. Fractional Differential Equations; Academic Press: New York, NY, USA, 1999.

33. Miller, K.S.; Ross, B. An Introduction to the Fractional Calculus and Fractional Differential Equations; Wiley: New York, NY, USA, 1993.

34. Kilbas, A.A.; Srivastava, H.M.; Trujillo, J.J. Theory and Applications of Fractional Differential Equations: Elsevier: Amsterdam, The Netherlands, 2006.

35. Samko, S.G.; Kilbas, A.A.; Marichev, O.I. Fractional Integrals and Derivatives: Theory and Applications; Gordon and Breach: New York, NY, USA, 1993.

36. Oldham, K.B.; Spanier, J. The Fractional Calculus; Academic Press: San Diego, CA, USA, 1974.

37. Mahmudov, N.I.; Ahmadova, A.; Huseynov, I.T. A new technique for solving Sobolev type fractional multi-order evolution equations. arXiv 2021. arXiv:2102.10318.

38. Fernandez, A.; Kürt, C.; Özarslan, M.A. A naturally emerging bivariate Mittag-Leffler function and associated fractional-calculus operators, Comput. Appl. Math. 2020, 39, 200. [CrossRef]

39. Arnold, L.; Kliemann, W.; Oeljeklaus, E. Lyapunov exponents of Linear stochastic systems. In Lyapunov Exponents, Lecture Notes in Math. 1186; Arnold, L., Wihstutz, V., Eds.; Springer: New York, NY, USA, 1986; pp. 85-125.

40. Arnold, L.; Papanicolau, G.; Wihstutz, V. Asymptotic analysis of Lyapunov exponent and rotation number of the random oscillator and applications. SIAM J. Appl. Math. 1986, 46, 427-450. [CrossRef]

41. Huong, P.T.; Kloeden, P.E.; Son, D.T. Well-posedness and regularity for solutions of Caputo stochastic fractional differential equations in $L^{p}$ spaces. Stoch Anal. Appl. 2021, 1-15. [CrossRef] 